\title{
Measurements of Dielectric Parameters of Aviation Fuel at X-Band Frequencies Using Cavity Perturbation Technique
}

\author{
Sarita Sharma ${ }^{a^{*}}$, Dalver Kaur ${ }^{b}$ \\ ${ }^{a}$ Department of Electronics \& Communication Engineering I K Gujral Punjab Technical University, \\ Jalandhar, 144601, India \\ b Department of Electronics \& Communication Engineering I K Gujral Punjab Technical University, \\ Jalandhar, 144601, India
}

\begin{abstract}
Real and imaginary parts of complex permittivity of Aviation Turbine Kerosine (Hydro-fined) JetA-1 and Aviation Turbine Kerosine (Copper-Sweetened) JetA-1 are measured over the wide frequency range 8-12 GHz using X-band rectangular cavity resonator. The measurement technique uses the cavity perturbation technique. The fuel sample is filled in thin capillary tube of low loss material. It is then inserted through a sample hole at the centre of the broader side of the waveguide cavity resonator. The sample shifts the resonance frequency. The resonance frequency and shift in resonance frequency are measured using PNA Network Analyzer (AT E8362C). Validity of present measurement technique has been checked by measuring the dielectric properties of well-known dielectric materials (Plexiglas). Due to lack of experimental data in literature on dielectric parameters of Aviation fuels, it would be of great interest for the community to find its dielectric properties over wider frequency range. In addition the estimation of measurement error associated with this technique is also discussed.
\end{abstract}

Index Terms: Complex Permittivity, Dielectric Constant, Dielectric Loss, Dielectric material, Cavity Resonator

(C) 2016 Published by MECS Publisher. Selection and/or peer review under responsibility of the Research Association of Modern Education and Computer Science

\section{Introduction}

Dielectric materials have found significant applications in recent microwave and telecommunication systems. There is a need for better understanding of dielectric properties of material and their measurement techniques.

* Corresponding author. Tel.: 09988292611;

E-mail address: s_saritasharma@yahoo.com 
Dielectric properties of materials are one of the important parameters required for the characterization of material at microwave frequencies. Material characterization is essential for the proper section and implementation of a substance when used in industrial, scientific and medical applications. Dielectric properties cannot be measured directly; it is usually calculated via other measurable parameters such as transmission /reflection coefficients (s-parameters), propagation constant, etc. Measurement of these parameters requires very precise and accurate experimental approach associated with specific formulas. Accurate information for the dielectric properties is required to study the possible hazard of EM field [1]. The dielectric properties of materials are determined by its molecular structure, if the molecular structure changes its dielectric properties changes. Any single technique for complex permittivity measurement is not suitable over a wide range of frequency and complex permittivity. Numerous techniques for determination of dielectric properties of dielectric material have been developed [2-3]. In past various techniques in both the frequency and time domains have been used to measure the dielectric properties of materials at microwave frequencies [5-9]. Among various frequency domain methods for the measurements of complex permittivity, cavity perturbation technique is one of the most widely used because of its relative simplicity for precise microwave measurements of conductivity, dielectric, and magnetic properties of materials. In this technique one measures the adiabatic change of the characteristics of a resonator upon the introduction of a foreign body (sample). This technique requires the accurate determination of quality factor and resonant frequency of microwave cavity resonator. Different cavity perturbation techniques have different limitations i.e the insertion of sample in the cavity [10]; size and shape of sample; type of cavity resonators etc. hence further progress in this field is required.

Normally, the samples required for measurements in a rectangular cavity are equal to the height of the cavity. When the samples are smaller than the height of the cavity, accurate measurements are often difficult [11]. The objective of cavity perturbation technique is to measure accurately and precisely the quality factor $Q$ and resonance frequency $f_{o}$ of unloaded and loaded microwave cavity resonator using transmission or reflection coefficient (s-parameters) as a function of frequency [1214]. Cavity perturbation techniques are applied to the study of low-loss and medium loss materials. However, the cavity perturbation technique presented here is applicable to all types of materials.

In petroleum industries accurate values of dielectric constant and dielectric loss of petroleum and hydrocarbon contaminated soil (petroleum based) are required to be known for their electromagnetic characterization [15]. The data of complex permittivity of petroleum based aviation fuel at X-band are not available in the literature. Hence, these measurements have been carried out to determine dielectric properties of aviation fuels at X-band by using Cavity perturbation method. In this paper the results of the experimentally measured dielectric constant $\left(\varepsilon^{\prime}\right)$ and loss factor $\left(\varepsilon^{\prime \prime}\right)$ of two types of petroleum based aviation fuels at X-band of microwave spectrum. The measurement setup uses cavity resonator and VNA. The real and imaginary parts of the complex permittivity have been calculated from the shift in the resonance frequency and change in the Q-factor. In order to verify the validity of the measurement technique, dielectric constants of standard dielectric material (Plexiglas) are measured at X-band and the measured vales are compared with the values available in literature [16]. However the dielectric data of presented aviation fuels over the entire frequency range of 8-12 $\mathrm{GHz}$ are not available for comparison.

\section{Measurement Technique}

Initially Cavity perturbation technique was proposed by Bethe and Schwinger [4]. Thereafter many researchers have reported theoretical and experimental analysis of cavity perturbation techniques [6-20]. The measurements of complex permittivity $\left(\varepsilon^{*}\right)$ and permeability $\left(\mu^{*}\right)$ are performed by inserting a small, 
appropriately shaped sample into a cavity and determining the properties of the sample from resultant change in the resonant frequency and loaded quality factor. The basic idea of the cavity perturbation is the change in the overall geometric configuration of the electromagnetic fields upon the insertion of a small sample must be small. Based on this assumption, a detailed derivation of the perturbation equation for the frequency shift upon the insertion of a sample into a cavity was given by Harrington [5]. The cavity perturbation technique described in this paper is based on Waldron [19]. Detailed analysis of present technique can be found in [17].

\subsection{Measurement Setup}

A transmission type rectangular cavity resonator is designed and fabricated using standard WR-90 waveguide at X-band with inner dimension of $23 \times 10 \mathrm{~mm}$. Depending upon the modes to be propagate, the length has been chosen. The fundamental mode is TE type with $10 n$ where $n$ is the number of half wavelength along the propagation direction. Two thin conducting sheets are used to close the two ends of the waveguide to form a cavity resonator. The inductive coupling is provided with two symmetric holes of diameter $4 \mathrm{~mm}$ on these end sheets. The design specifications are given below.

Frequency Range: $8 \mathrm{GHz}-12 \mathrm{GHz}$ (X-band)

Cut of wavelength: $46 \mathrm{~mm}$

Modes Propagates: $5\left(\mathrm{TE}_{105}, \mathrm{TE}_{106}, \mathrm{TE}_{107}, \mathrm{TE}_{108}, \mathrm{TE}_{109}\right)$

Inner dimension of cavity: $22.9 \times 10 \times 140 \mathrm{~mm}^{3}$

Outer dimension of cavity: $25 \times 12 \times 140 \mathrm{~mm}^{3}$

Material used: Brass

Coupling hole: $4 \mathrm{~mm}$

In order to insert a sample material in the resonator cavity without disassembling it, a narrow hole is constructed at the centre of the broader side of the cavity. The narrow opened hole has negligible effect on changing the geometrical configuration of electromagnetic field inside the cavity. The width of the sample hole is equal to the diameter of the cylindrical sample. Fuel sample filled in a thin capillary tube of very low loss material is kept at the centre of the broader side of the cavity resonator through this sample hole. This rectangular waveguide cavity resonator is connected to two ports of the Network Analyzer for S-parameters measurement. A conventional Thru-reflect-line (TRL) calibration technique is applied for calibration. The cavity resonator has multiple resonant frequencies in particular frequency band, TRL calibration was reused in each resonant frequency range and the measurement was separately performed in each resonant frequency range.

When fuel sample is inserted in the cavity, it will cause shift in resonant frequency and $Q$-factor of empty cavity. Resonance peaks occur at different resonance frequencies $\left(f_{0}\right)$ for different modes of the cavity resonator. When the volumes of the cavity resonator and the sample, the resonant frequencies $\left(f_{0} \& f_{s}\right)$ and quality factors $\left(Q_{0} \& Q_{s}\right)$ before and after the sample insertion are measured, the real part $\left(\varepsilon^{\prime}\right)$ and imaginary part $\left(\boldsymbol{\varepsilon}^{\prime \prime}\right)$ of the complex permittivity of the sample can be calculated using equations (1) \& (2)

$$
\begin{aligned}
& \varepsilon^{\prime}=\frac{V_{c}\left(f_{0}^{2}-f_{s}^{2}\right)}{4 V_{s} f_{s}^{2}} . \\
& \varepsilon^{\prime \prime}=\left(\frac{1}{Q_{s}}-\frac{1}{Q_{0}}\right) \frac{V_{c} f_{0}^{2}}{4 V_{s} f_{s}^{2}} .
\end{aligned}
$$


where $V_{\mathrm{c}}$ is volume of the cavity and $V_{\mathrm{s}}$ is the volume of the sample. $Q_{\mathrm{s}}$ is the quality factor of cavity loaded with sample and $Q_{0}$ is the quality factor without sample. The measured values of $\mathrm{Q}\left(Q_{0} \& Q_{s}\right)$ of the cavity are calculated by the equation given below.

$$
Q=\frac{f_{\text {resonant }}}{\Delta f_{3 d B}}=\frac{f}{f_{R}(3 d B)-f_{L}(3 d B)} .
$$

Equation (1) and (2) are the expression for complex permittivity using cavity perturbation technique [17].

\subsection{Error analysis}

No measurement of a physical quantity can be entirely accurate. There is an inherent error margin associated with the results. Error in measurement is the difference between the measured value and the unknown, true, value of the measured quantity [20]; $\sigma_{\text {error }}^{2}=\sigma_{\text {Measurement }}^{2}-\sigma_{\text {True. }}^{2}$

The precision of measurement can best be improved through the correction of the causes of variation in the measurement process. However it is frequently desirable to estimate the confidence interval for the mean of measurements which includes the measurement error variation. The confidence interval for the mean of these measurements is reduced by obtaining multiple readings. To estimate the total measurement error, uncertainty analysis is conducted on the present measurements. The calculation of uncertainty requires a detailed budget which breaks down the variance of measurement error into consistent components, each of which can be separately estimated. The detailed model becomes like: $\sigma_{M}^{2}=\sigma_{\text {instrument }}^{2}+\sigma_{\text {fixture }}^{2}+\sigma_{\text {enivironment }}^{2}+\sigma_{\text {calibration }}^{2}$ $+\sigma_{\text {sample }}^{2}+\sigma_{\text {analysis. }}^{2}$

Uncertainty due to repeatability i.e. series of observations / readings at different interval of time, variations in cables, connectors, relevant accessories, AC mains, change of operators, variations in environment conditions i.e. temp, humidity etc. have been calculated by taking standard deviation of the mean of the readings of five observations of each parameters. Uncertainty due to standard deviation (Usd) is calculated as follows:

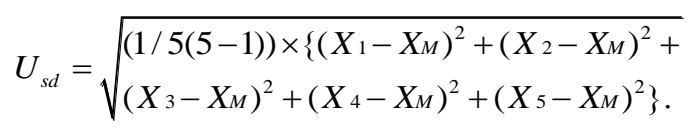

where $X_{1}, X_{2}, X_{3}, X_{4}, X_{5}$ are readings at different interval of time and $X_{M}$ is mean value. The degree of freedom $=($ No. of observations $)-1$ i.e. $5-1=4$.

Uncertainty arises from flaw in the measurement which repeats each time a measurement is made. It is mainly due to errors in the calibration of the measuring instruments. It depends upon calibrator's accuracy; its resolution; calibration certificate, and its temperature drift specifications etc. Uncertainty due to Calibrator's accuracy $\left(\mathrm{U}_{\mathrm{a} 1}\right)$, Calibrator's resolution $\left(\mathrm{U}_{\mathrm{a} 2}\right)$, Calibrator's Calibration Certificate $\left(\mathrm{U}_{\mathrm{a} 3}\right)$, and due to Temp Drift in Calibrator's Specifications $\left(\mathrm{U}_{\mathrm{a} 4}\right)$ at $95 \%$ Confidence is calculated. Combined uncertainty $(\mathrm{Uc})$ will be

$$
U c=\sqrt{\left(U_{s d}\right)^{2}+\left(U_{a 1}\right)^{2}+\left(U_{a 2}\right)^{2}+\left(U_{a 3}\right)^{2}+\left(U_{a 4}\right) ?}
$$

Overall Measurement Uncertainty $\pm U=k U c$; where $k=1.96$ for $95 \%$ confidence level. At $95 \%$ confidence interval, the error is $\pm 1.5 \mathrm{ppm}$. 


\section{Results and Discussion}

The cavity was connected to a network analyzer and excited to operate in five modes. Accordingly, five resonant peaks corresponding to frequencies $\left(f_{0}\right)$ around $8.46,9.15,9.96,10.7$ and $11.64 \mathrm{GHz}$ appeared on the screen of the analyzer. When the cavity is loaded with fuel sample, shift in resonant frequencies and $Q$-factors of empty cavity occur. Resonance peaks occur at different resonance frequencies $\left(f_{s}\right)$ for different modes of the cavity resonator. The S-parameter (transmission co-efficient) measurement shows the expected shifts in resonance frequency and quality factor. $3 \mathrm{~dB}$ down point on the resonance curves are noted down to calculate quality factor $\left(Q_{0}\right)$ of empty cavity and sample loaded cavity $\left(Q_{s}\right)$. Using equations (1) \& (2), dielectric constant and dielectric loss of sample materials have been calculated.

The dielectric constant and dielectric loss of Plexiglas are measured at X-band. Table 1 shows the measured values of dielectric constant of Plexiglas. It is observed from table 1 that measured dielectric constant $\left(\boldsymbol{\varepsilon}^{\prime}\right)$ is very close to available data in literature; $\boldsymbol{\varepsilon}^{\prime}=2.59$ [21] \& $\boldsymbol{\varepsilon}^{\prime}=2.65$ at $10 \mathrm{GHz}$ (free space method) [16].

Table 1. Measured vales of dielectric constant of Plexiglas

\begin{tabular}{ccc}
\hline $\left.\mathrm{f}_{0} \mathrm{GHz}\right)$ & $\mathrm{f}_{\mathrm{S}}(\mathrm{GHz})$ & Dielectric Constant $\left(\varepsilon^{\prime}\right)$ \\
\hline 8.46 & 8.26 & 2.17 \\
9.15 & 8.91 & 2.41 \\
9.96 & 9.67 & 2.69 \\
11.64 & 11.29 & 2.78 \\
\hline
\end{tabular}

Complex permittivity of different samples of Aviation fuels are measured at X-band (8-12 GHz). The shift in resonance frequency \& Q-factor depend on the dielectric properties of the material; the dielectric constant and dielectric loss of both samples of fuels are measured on that specific frequency. In present measurement the shift in resonance frequency for both Aviation fuels varies from $8.27 \mathrm{GHz}$ to $11.44 \mathrm{GHz}$. Measured values of dielectric constant $\left(\varepsilon^{\prime}\right)$ and loss factor $\left(\varepsilon^{\prime \prime}\right)$ for Kerosine (copper sweetened) Jet A-1 and Turbine Kerosine (hydrofined) Jet A-1 at $8.46 \mathrm{GHz}, 9.15 \mathrm{GHz}, 9.96 \mathrm{GHz}$ and11.64 GHz are presented in Fig. 1 and Fig. 2 respectively as a function of frequency.

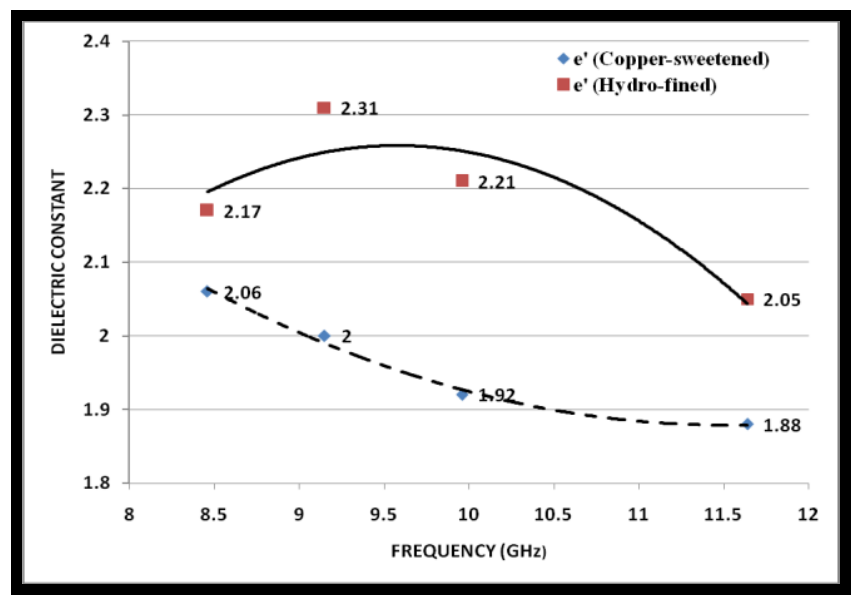

Fig.1. Frequency dependence of Dielectric Constant of Aviation fuels 


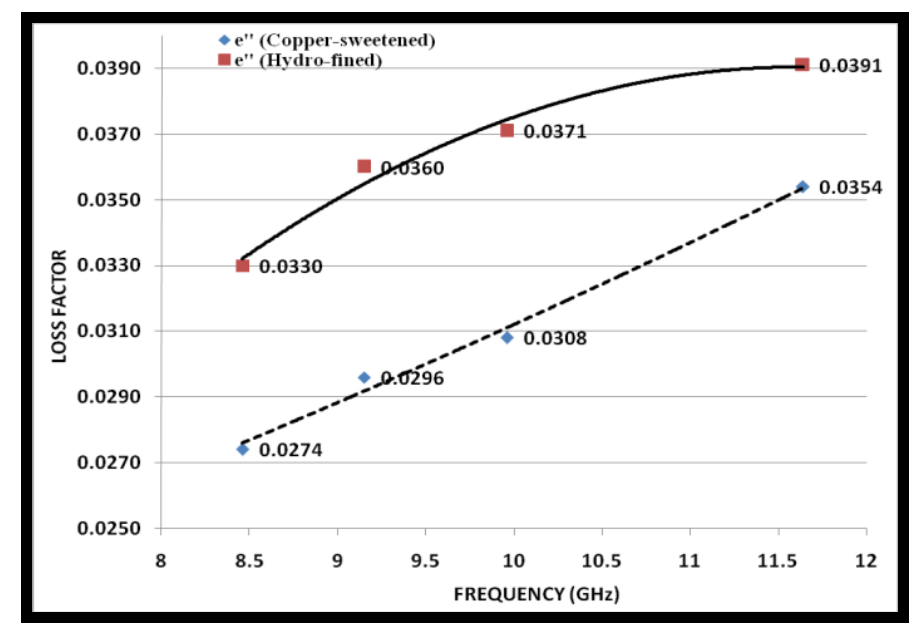

Fig.2. Frequency dependence of Loss factor of Aviation fuel

Least-square method and polynomial interpolation of measured values for $\varepsilon^{\prime}$ and $\varepsilon^{\prime \prime}$ in this work satisfy the following empirical equations

$$
\begin{aligned}
& \boldsymbol{\varepsilon}^{\prime}{ }_{\text {(hydro-fined) }}=-0.052 f_{0}{ }^{2}+1.010 f_{0}-2.579 \\
& \boldsymbol{\varepsilon}^{\prime}{ }_{\text {(copper-sweetened) }}=0.019 f_{0}{ }^{2}-0.443 f_{0}+4.442 \\
& \boldsymbol{\varepsilon}^{\prime \prime}{ }_{\text {(hydro-fined) }}=0.013 f_{0}-0.040 \\
& \boldsymbol{\varepsilon}^{\prime \prime}{ }_{\text {(copper-sweetened) }}=6 \mathrm{E}-05 f_{0}{ }^{2}+0.001 f_{0}+0.012
\end{aligned}
$$

Above empirical equations can be used to measure the complex permittivity of Aviation fuels under test at other desired frequency

\section{Conclusions}

Cavity perturbation technique for measuring the complex permittivity of two types of Aviation fuels over wide frequency range of $\mathrm{X}$-band $(8-12 \mathrm{GHz})$ is described here. Accuracy of measurement technique is checked by measuring the dielectric constant of Plexiglas. Measured results have been compared with those existing in literature to show good agreement. It is observed from fig. $1 \& 2$ that the values of dielectric constant for both Aviation fuels are decreasing with increase of frequency whereas loss factor increase with increase of frequency. It is also observed that Aviation Turbine Kerosine (Hydro-fined) JetA-1 is more lossy then Aviation Turbine Kerosine (Copper-Sweetened) JetA-1 at same frequencies. For Aviation fuels, the dielectric data over entire frequency range are not available, so the dielectric properties of Aviation fuels presented here is of great interest for the community. Measurement error has been estimated and found that at $95 \%$ confidence interval, the error is $\pm 1.5 \mathrm{ppm}$. The dielectric properties of the material can be measured with existing cavity resonator with an accuracy of $1.5 \mathrm{ppm}$.

\section{Acknowledgements}

Authors acknowledge Microwave Laboratory at Bharat Electronics for measurement purposes in this work. 


\section{References}

[1] A. Lonappan, G. Bindu, V. Thomas, and K. T. Mathew, "Analysis of Human Semen Using Microwaves," Progress In Electromagnetics Research, PIER 57, pp. 277-284, 2006.

[2] H.E. Bussey, "Measurement of RF properties of materials - a survey," Proc. IEEE, Vol. 55, No 6, pp. 1046-1053, 1967.

[3] A. Kraszewski, Microwave aquametry - a review, J. Microwave Power \& Electromag. Energy, Vol. 15, No 4, pp. 209-220, 1980.

[4] H. A Bethe and J. Schwinger, NRDC Report D1-117, Cornell University, March 1943.

[5] R. F. Harrington, Time-Harmonic Electromagnetic Fields, McGraw-Hill, New York, 1961.

[6] B. Viswanathan and R. Raman, N. S. Raman and V. R. K. Murthy, Microwave power loss and XPS measurements on high $\mathrm{T}_{\mathrm{c}} \mathrm{Nd}$---Ba---Cu---Oxide superconducting system, Solid State Communication, vol. 66, no.4, pp. 409-411, 1988.

[7] Y. Xu and R. G. Bosisio, Analysis of different coaxial discontinuities for microwave permittivity measurements, IEEE Trans. Instrum. Meas., vol. 42, pp. 538-543, April 1993.

[8] M.S. Venkatesh and G.S.V. Raghavan, "An overview of dielectric properties measuring techniques" Canadian Biosystem Engineering, vol 47, 2005.

[9] Mi Lin, Mohammed Afsar, "A new cavity perturbation technique for accurate measurement of dielectric parameters" IEEE MTTS International Microwave Symposium Digest (2006).

[10] Ikeda, M.; Fukunaga, T., "Miura, T, Influence of sample insertion hole on resonant cavity perturbation measuring method," Microwave Symposium Digest, 2003 IEEE MTT-S International, vol. 2, pp. 1423 1426.

[11] Verma, A., "Dube, D.C, Measurement of dielectric parameters of small samples at X-band frequencies by cavity perturbation technique," Instrumentation and Measurement, IEEE Transactions, Vol. 54, Issue 5, pp. 2120 - 2123, Oct. 2005.

[12] J. Baker-Jarvis, E.J. Vanzura, W.A. Kissick, "Improved technique for determining complex permittivity with transmission/ reflection method," IEEE Trans. Microwave Theory Tech., Vol. 38, No 8, pp.10961103, 1990.

[13] Paul J. Petersan and Steven M. Anlagea, "Measurement of resonant frequency and quality factor of microwave resonators: Comparison of methods" Journal of Applied Physics, Vol. 84, No. 6, pp. 33923402, 15 September 1998.

[14] M.D. Janezic, and J.A. Jargon, Complex permittivity determination from propagation constant measurements, IEEE Microwave and Guided Wave Letters, Vol. 9, No 2, pp.76-78, 1999.

[15] Chenhui Liu et al "Temperature and Moisture Dependence of the Dielectric Properties of Silica Sand" Journal of Microwave Power and Electromagnetic Energy, 47 (3), pp. 199-209, 2013.

[16] D.K Ghodgaonkar, V.V. Varadan and V. K. Varadan, "A Free Space Method for Measurement of Dielectric Constant and Loss Tangents at Microwave Frequencies," IEEE Transaction on Instrumentation and Measurement, vol. 38, pp. 789 - 793, 1989.

[17] Sarita Sharma et al, "Measurement of Dielectric Constant and Loss Factor of The Dielectric Material at Microwave Frequencies," Journal of Electromagnetic Waves \& Applications, MIT USA, PIERS 69, pp 47-54, 2007.

[18] V. R. K. Murthy and R. Raman, "A method for the evaluation of microwave dielectric and magnetic parameters using rectangular cavity perturbation technique," Solid State Communication, vol. 70, no. 8, pp. 847-850, 1989.

[19] Waldron, R. A., "Perturbation theory of resonant cavities," Proc. IEE, vol. 170C, pp. 272-274, 1960.

[20] Grant, E.L and Leavenworth, R.S. Statistical Quality Control, 6th edition, McGraw-Hill, New York, 1988 
[21] Von Hippel, A. R., Dielectric Materials and Applications, John Wiley \& Sons, New York, 1954.

\section{Authors' Profiles}

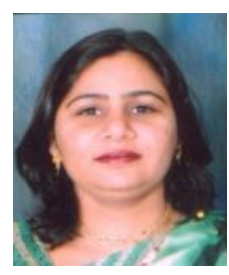

Sarita Sharma received first class B.Tech and M.Tech degree in Electronics and Communication Engineering from PTU, Jalandhar (Punbaj), India. She worked as faculty in department of Electronics Technology, Guru Nanak Dev University, Amritsar, India. Thereafter she joined as Asst. Professor in department of ECE at Chandigarh College of Engineering and Technology, Chandigarh, India. She is research scholar in the department of Electronics \& Communication Engineering, Punjab Technical University, Jalandhar. Her research interests are in the area of microwave passive devices, RF communication systems, and microwave measurements.

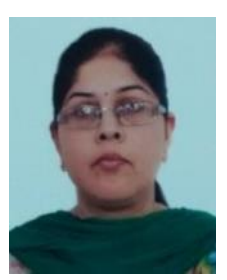

Dalveer Kaur received the first class M.Tech. degree in Microelectronics in 2003 and the Ph.D. degree in Electronics \& Microwave Engineering in 2010 from Guru Nanak Dev University, Amritsar, India. Since 2010 she is working as Asst. professor in the department of Electronics \& Communication Engineering, Punjab Technical University, Jalandhar, India. Her research interests are in the area of microwave communication, microwave devices, and microwave measurements. communication, microwave devices, and microwave measurements

How to cite this paper: Sarita Sharma, Dalver Kaur,"Measurements of Dielectric Parameters of Aviation Fuel at X-Band Frequencies Using Cavity Perturbation Technique", International Journal of Wireless and Microwave Technologies(IJWMT), Vol.6, No.6, pp.48-55, 2016.DOI: 10.5815/ijwmt.2016.06.05 